\title{
Tocotrienols for normalisation of hepatic echogenic response in nonalcoholic fatty liver: a randomised placebo-controlled clinical trial
}

Enrico Magosso', Mukhtar Alam Ansari ${ }^{1}$, Yogheswaran Gopalan 2,3, Ibrahim Lutfi Shuaib ${ }^{1}$, Jia-Woei Wong ${ }^{4}$, Nurzalina Abdul Karim Khan ${ }^{2}$, Mohamed Rizal Abu Bakar ${ }^{1}$, Bee-Hong Ng${ }^{4}$ and Kah-Hay Yuen ${ }^{2 *}$

\begin{abstract}
Background: Nonalcoholic fatty liver disease (NAFLD) is one of the commonest liver disorders. Obesity, insulin resistance, lipid peroxidation and oxidative stress have been identified amongst the possible hits leading to the onset and progression of this disease. Nutritional evaluation of NAFLD patients showed a lower-than-recommended intake of vitamin E. Vitamin E is a family of 8 isoforms, 4 tocopherols and 4 tocotrienols. Alpha-tocopherol has been widely investigated in liver diseases, whereas no previous clinical trial has investigated tocotrienols for NAFLD. Aim of the study was to determine the effects of mixed tocotrienols, in normalising the hepatic echogenic response in hypercholesterolaemic patients with ultrasound-proven NAFLD.
\end{abstract}

Methods: Eighty-seven untreated hypercholesterolaemic adults with ultrasound-proven NAFLD were enrolled and randomised into control group $(n=44)$ and tocotrienols group $(n=43)$. The treatment, either mixed tocotrienols $200 \mathrm{mg}$ twice daily or placebo, had a 1-year duration.

Normalisation of hepatic echogenic response, being the trial primary aim, was used in sample size calculations. The data were assessed according to intention to treat principle as primary outcome. Per protocol analysis was also carried out as secondary outcome measurement.

Results: Thirty and 34 participants concluded the study in the tocotrienols and placebo group respectively. Alpha-tocopherol levels were within the normal range for all subjects. As primary outcome, the normalisation of hepatic echogenic response was significantly higher for the tocotrienols treated group compared to the placebo group in the intention to treat analysis ( $P=0.039 ; 95 \% \mathrm{Cl}=0.896-6.488)$. As secondary objective, the per protocol assessment also showed significant rate of remission $(P=0.014 ; 95 \% \mathrm{Cl}=1.117-9.456)$. Worsening of NAFLD grade was recorded in two patients in the placebo group, but none in the group treated with tocotrienols. No adverse events were reported for both groups.

Conclusion: This is the first clinical trial that showed the hepatoprotective effects of mixed palm tocotrienols in hypercholesterolemic adults with NAFLD.

Trial registration: Clinicaltrials.gov, NCT00753532.

\footnotetext{
* Correspondence: khyuen@usm.my

${ }^{2}$ School of Pharmaceutical Sciences, Universiti Sains Malaysia, Penang, Malaysia

Full list of author information is available at the end of the article
}

\section{Biomed Central}

(c) 2013 Magosso et al.; licensee BioMed Central Ltd. This is an open access article distributed under the terms of the Creative Commons Attribution License (http://creativecommons.org/licenses/by/2.0), which permits unrestricted use, distribution, and reproduction in any medium, provided the original work is properly cited. 


\section{Background}

In recent years research on non-alcoholic fatty liver disease (NAFLD) has been intensified indicating an increased interest in this common hepatic disorder. Lipid peroxidation and oxidative stress have been recognised as playing a pivotal role in the pathogenesis and progression of NAFLD [1,2]. Nutritional assessment of NAFLD patients showed a lower-than-recommended intake of vitamin E [3], possibly leading to low plasma concentration of antioxidants. Recent clinical studies suggested vitamin $\mathrm{E}$ to be one of the most promising agent for amelioration of non-alcoholic steatohepatitis (NASH) in adults [4] and up to a certain extent in youngsters [5]. The most common homologue of the vitamin E family investigated is alpha-tocopherol. Other homologues of vitamin E, namely tocotrienols, also exist in isomers designated as alpha, beta, gamma and delta, and they differentiate from analogous isomers of tocopherol by the presence of an unsaturated phytyl chain ('tail') as opposed to a saturated one in tocopherols.

Tocotrienols are preferentially distributed to the liver $[6,7]$ and alpha-tocotrienol has been reported to be 40 60 times more potent than alpha-tocopherol against lipid peroxidation in rat liver microsomes [8].

Prevalence of NAFLD in Asia has been showed in a recent review to be similar to Western countries [9], with local data in hypercholesterolaemic adults indicating a NAFLD prevalence of about 60\% [10]. Concerns about the high prevalence of NAFLD and the not fully understood reasons for its progression to more severe form of liver diseases, have led to the initiation of this interventional, double-blind placebo-controlled study. The aim of the investigation was to evaluate the activity of tocotrienols in normalising hepatic echogenic response in adults with NAFLD with hypercholesterolaemia as an associated risk factor.

\section{Methods}

\section{Patients}

Volunteers of both genders aged 35 years and above with mild untreated hypercholesterolaemia and ultrasound-proven NAFLD, selected from a population-based study on the prevalence of NAFLD [10] were assessed for eligibility. Mild hypercholesterolemia, assessed during screening, was defined as ranging between 5.2 and $6.2 \mathrm{mmol} / \mathrm{L}(200-240 \mathrm{mg} / \mathrm{dL})$ for total cholesterol and between $2.6 \mathrm{mmol} / \mathrm{L}$ and $4.2 \mathrm{mmol} / \mathrm{L}(100-161 \mathrm{mg} / \mathrm{dL})$ for LDL-cholesterol. Alcohol intake, assessed through interview, had to be less than $20 \mathrm{~g} /$ day. Alanine transaminase (ALT), aspartate transaminase (AST) and gamma-glutamyl transpeptidase (GGT) had to be below 3 times the respective upper limit value of $53 \mathrm{IU} / \mathrm{L}, 40 \mathrm{IU} / \mathrm{L}$ and $49 \mathrm{IU} / \mathrm{L}$ for male or $32 \mathrm{IU} / \mathrm{L}$ for female.
Volunteers were excluded if they were presented in the 3 months prior to enrolment with anti-hyperlipidemic treatment and/or vitamin E intake and history of abuse or excessive intake of alcohol, previous cardiovascular events or hepatitis. No specific dietary guidance was given to the participants, however they were advised about the overall health benefits of increased physical activity and fat-reduced diet.

Participants were recruited at Universiti Sains Malaysia facilities in Kepala Batas Hospital (Penang, Malaysia), between February 2008 and June 2009. Out of 102 hypercholesterolaemic adults with ultrasound-proven NAFLD assessed for eligibility, 87 fulfilled the inclusion criteria and were enrolled by the investigators EM and YG, who also dispensed the treatments.

The present interventional study (http://www.clinicaltrials.gov/show/NCT00753532), designed as a parallel double-blind placebo-controlled study, was approved by the Research Ethics Committee for Human Studies of Universiti Sains Malaysia. Participants were enrolled upon signing informed consent.

\section{Randomisation and treatment}

The treatment assigned to participants for 1 year was either mixed tocotrienols $200 \mathrm{mg}$ twice daily or placebo. The content in each capsule of the mixed tocotrienols preparation was $61.5 \mathrm{mg}, 112.8 \mathrm{mg}$ and $25.7 \mathrm{mg}$ for alpha-, gamma- and delta-tocotrienol, respectively and $61.1 \mathrm{mg}$ of alpha-tocopherol. Both placebo and tocotrienols, were soft gel capsules and were similar in terms of colour, size, shape and surface texture. Participants were randomised using a computer generated random allocation sequence. A permuted block design was employed. Each block of specified size contained allocation ratio of 1:1 (placebo:tocotrienols). The random allocation sequence would select the next block and determine the next allocations. Sequence was not made known to researchers who enrolled participants. For the purpose of assigning participants to interventions, a subject number was used. The researcher (WJW) who generated the random allocation sequence and assigned participants was blinded to subjects' clinical data and was independent from the persons who enrolled participants.

The mixed tocotrienols preparation (Tocovid Suprabio ${ }^{\circledR}$ ) and the placebo capsules were purchased from Hovid (Ipoh, Malaysia). Researchers and volunteers were blinded to the assigned treatment.

\section{Clinical and metabolical evaluation}

Quarterly, after an overnight fast, a $12 \mathrm{ml}$ blood sample from each volunteer was withdrawn and analysed by an accredited laboratory for levels of serum total cholesterol (TC), LDL-cholesterol, HDL-cholesterol, triglycerides (TG), apolipoprotein B (ApoB), lipoprotein A (LP(A)), 
high-sensitivity C-reactive protein (hs-CRP), fasting glucose, serum creatinine, alkaline phosphatase (ALP), ALT, AST and GGT. Body mass index (BMI) and blood pressure were measured. Moreover, participants were asked to recall their lifestyle habits (such as food intake and physical activity). Compliance to the study treatment was assessed through patient recall, capsule count and plasma levels of tocotrienols. Plasma tocotrienol concentrations were measured using a validated highperformance liquid chromatographic (HPLC) method [11] after completion of the study, until then the samples were kept at $-80^{\circ} \mathrm{C}$. Quantitative HPLC analysis of plasma tocotrienol concentrations were performed to determine adherence to the treatment. Investigation of the association of plasma level of tocotrienols with response was not the aim of the present study.

High sensitivity B-mode ultrasonography (USG) examination was performed at baseline and after 1 year by the same experienced radiologists (MAA and ILS, in presence of a third radiologist, MRAB) using the same instrument, a Pentax-Hitachi EUB6500 (Tokyo, Japan) fitted with a EUP-C516 (3.5-5.0 MHz) probe using the standard adult abdominal settings, throughout the study. The radiologists were unaware of the clinical, metabolic and USG baseline conditions of the subjects. The USG evaluation consisted of a visual scoring system evaluating three aspects of interest: hepatorenal echodiscrepancy, posterior echo-penetration and portal vein wall clarity. Each of the three hepatic aspects considered was given a score of zero, if normal, 1 or 2 according to the echogenicity of the response [12]. Hepatic aspects and relative scoring system are detailed in Table 1 . The values were then summed up and a total score of 3 and

\begin{tabular}{l} 
Table 1 Criteria of the hepatic aspects considered and \\
relative scoring system for ultrasonographic diagnosis of \\
nonalcoholic fatty liver disease \\
\hline Criteria
\end{tabular}

Liver/kidney parenchyma

a) Homogeneous echotexture and absence of significant contrast with kidney parenchyma

b) Slight increase in liver/kidney echodiscrepancy

C) Extreme echodiscrepancy between liver and kidney

Portal vein walls clarity

a) Clear definition of portal vein walls and structures

b) Decreased definition of portal vein walls and structures

C) Blurred visualisation of portal vein walls

Posterior echo-penetration

a) Clear definition of hepatic structures from diaphragm

b) Decreased definition of liver and diaphragm structures

c) Blurred diaphragm with loss of definition

(Adapted from [12]). above was diagnosed as fatty liver. Steatosis was graded as mild (score 3), moderate (score 4) or severe (score 5-6). Only diffuse hyperechogenicity of the liver parenchyma was acceptable, whereas focal or patchy responses were excluded.

\section{Statistical analysis}

Prospective estimation of the sample size for a binary outcome trial with 5\% significance level (alpha) and 80\% power was calculated according to Pocock [13]. Therefore, the minimum number of patients required per arm was calculated to be 20 . The binary outcomes considered were normalisation of echogenic response versus noimprovement or worsening.

Difference in proportion of participants that have been found with normal echogenic response at conclusion of the study between each group of treatment were assessed using Helmert-Pearson's Chi-square test. The effects of independent variables, such as blood parameters and anthropometric data, were evaluated using ANOVA for split-plot design adjusted for unweighted-means [14]. Homogeneity of baseline characteristics was calculated using Wilcoxon Rank Test. Statistical calculations were performed using Numbers'09 (Apple, Cupertino, CA, USA) and QuickCalcs or InStat 3.1a for Macintosh (GraphPad Software, San Diego California USA).

The study primary end-point was a normalisation of hepatic echogenic response, defined as homogeneous hepatic echotexture with absence of significant contrast with kidney parenchyma, clear definition of portal vein walls and clear definition of hepatic structures from diaphragm (Table 1). The data were assessed according to intention to treat and per protocol analyses. Drop-out patients, as well as those with worsened condition, were computed as non-responders.

\section{Results \\ Patients characteristics}

The study participants self-reported mostly sedentary lifestyles, with none of them engaged in regular physical exercise of minimum 30 minutes 3-time weekly. Out of 87 enrolled patients 64 completed the investigation. In the tocotrienols group 8 subjects withdrew consent, 3 were excluded for protocol violation and 2 did not come for the final USG follow-up. Similarly, in the placebo group 6 subjects withdrew consent, 2 were excluded for protocol violation and 2 did not come for the final USG follow-up. Initiating anti-hyperlipidemic therapy was the protocol violation that led to the exclusion of 5 participants from the study. Flowchart of the study is shown in Figure 1.

Overall, mean baseline characteristics of the 87 enrolled subjects were aged $51 \pm 8$ years (ranging from 36 to 74 years), BMI $27.2 \pm 4.6 \mathrm{~kg} / \mathrm{m}^{2}$, TC $5.7 \pm 0.6 \mathrm{mmol} / \mathrm{L}$, 


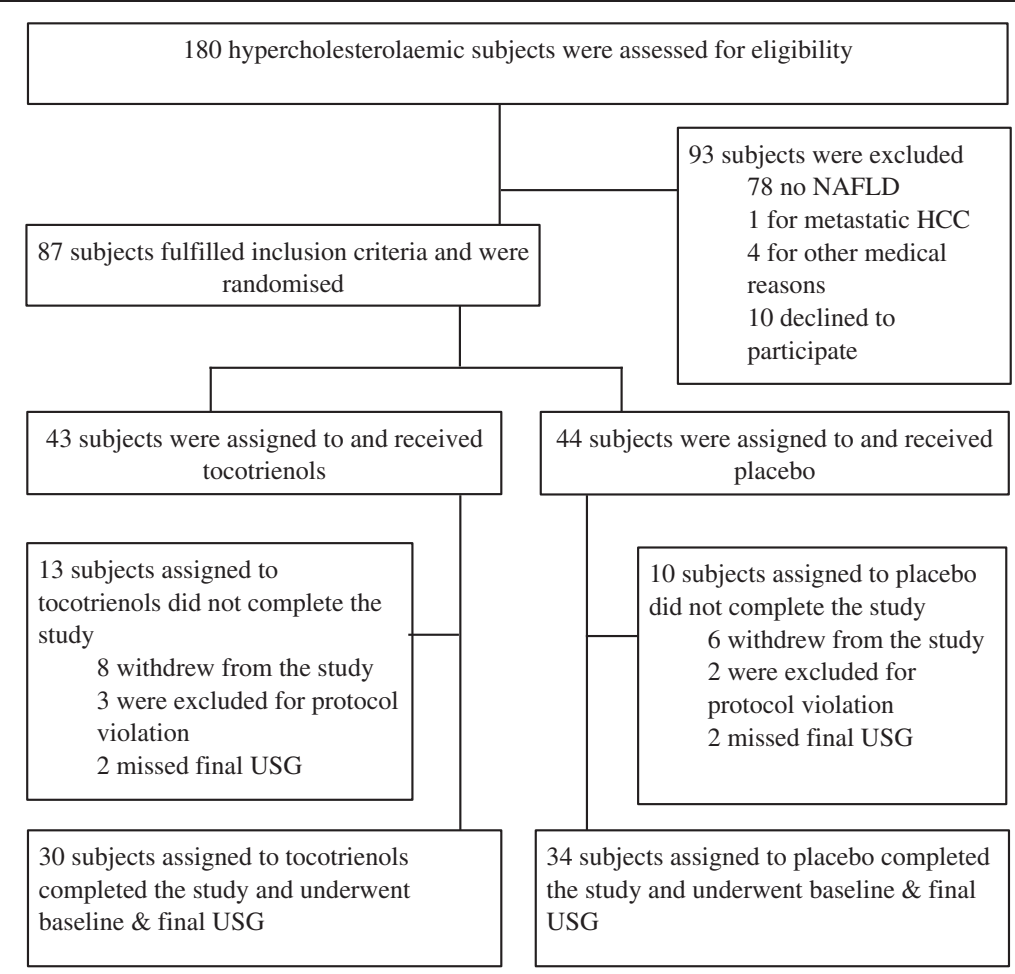

Figure 1 CONSORT Flow-chart of the clinical trial, with reason for exclusion of assessed subjects and for subjects that did not concluded the study. HCC = Hepatocellular carcinoma; USG = ultrasonography examination.

AST and ALT $37 \pm 13 \mathrm{IU} / \mathrm{L}$ and $37 \pm 19 \mathrm{IU} / \mathrm{L}$, respectively. Thirty subjects (34.5\%) were normoweight (BMI below 25), 37 (42.5\%) overweight (BMI ranging between 25 and 30), 19 (21.8\%) obese (BMI between 30 and 40) and only 1 (1.1\%) subject was morbidly obese (BMI between 40 and 50). Nine subjects $(10.3 \%)$ had impaired fasting glucose (IFG) above $7.0 \mathrm{mmol} / \mathrm{L}$. Mild fatty liver was the most common finding with 69 (79.3\%) cases, while moderate and severe fatty liver were found in $15(17.2 \%)$ and 3 (3.4\%) subjects, respectively. Ten participants (11.5\%) were smokers, equally distributed amongst tocotrienols (11.6\%) and placebo groups (11.4\%), respectively. The two groups of enrolled subjects were found to be homogeneous with regard to baseline characteristics (Table 2).

\section{Outcomes of USG hepatic evaluation}

As for primary objective (Table 3), applying the intention to treat analysis to all the 87 initially randomised subjects, 43 in the tocotrienols group and 44 in the placebo group, a statistically significant advantage was seen for tocotrienols versus placebo for the normalisation of hepatic echogenic response $(P=0.039$; Odds Ratio $[\mathrm{OR}]=$ 2.411; 95\% Confidence Interval $[\mathrm{CI}]=0.896-6.488$; Number Needed to Treat $[\mathrm{NNT}]=6$ ).

The secondary objective per protocol analysis of the subjects that concluded the study, thus underwent the final USG examination, showed that 15 out of 30 patients
$(50.0 \%)$ in the tocotrienols group were found with normal echogenic response compared to only 8 out of 34 patients (23.5\%) of the placebo group. Patients randomised to tocotrienols after 1 year of treatment presented a statistically significant normalisation of echogenic response compared to placebo $(P=0.014 ; \mathrm{OR}=3.250 ; 95 \% \mathrm{CI}=$ 1.117-9.456; NNT = 3.8).

Details of the clinical evaluation of subjects and relative changes after 1 year of treatment are presented in Table 4.

Worsening of steatotic grade was seen in two cases in the placebo group, but none in the tocotrienols group. Furthermore, amelioration of steatotic grade of more than one degree has been recorded in one male subject of the tocotrienols group, that improved from severe to mild and in two male subjects that improved from moderate to negative. Whereas no changes of more than one degree were observed in the placebo group.

\section{Blood biochemistry}

No statistically significant changes were seen amongst the two groups of subjects between baseline and conclusion of the study (Table 5) with regard to blood biochemistry results. Exception was ApoB, that showed a statistically $(P=0.046)$, but not clinically significant difference since the values were within the normal range. This difference was explained with gender difference for 
Table 2 Details of all subjects enrolled

\begin{tabular}{|c|c|c|c|}
\hline Variable & $\begin{array}{c}\text { Tocotrienols } \\
(n=43)\end{array}$ & $\begin{array}{l}\text { Placebo } \\
(n=44)\end{array}$ & $P$ value \\
\hline Gender (female) & $23(53.5 \%)$ & $30(68.2 \%)$ & 0.160 \\
\hline Age in years (range) & $52 \pm 9(36-74)$ & $49 \pm 7(38-68)$ & 0.177 \\
\hline $\mathrm{BMI}\left(\mathrm{kg} / \mathrm{m}^{2}\right)$ & $27.2 \pm 4.6$ & $27.1 \pm 4.6$ & 0.873 \\
\hline Normoweight (BMl < 25) & $16(37.2 \%)$ & $14(31.8 \%)$ & \\
\hline Overweight $(25$ < BMI < 30) & $16(37.2 \%)$ & $21(47.7 \%)$ & \\
\hline Obese $(30<\mathrm{BMI}<40)$ & $11(25.6 \%)$ & $8(18.2 \%)$ & \\
\hline Morbidly Obese $(40<\mathrm{BMI}<50)$ & $0(0.0 \%)$ & $1(2.3 \%)$ & \\
\hline $\mathrm{TC}(\mathrm{mmol} / \mathrm{L})$ & $5.9 \pm 0.6$ & $5.6 \pm 0.6$ & 0.075 \\
\hline $\mathrm{HDL}(\mathrm{mmol} / \mathrm{L})$ & $1.44 \pm 0.35$ & $1.37 \pm 0.30$ & 0.611 \\
\hline LDL (mmol/L) & $3.7 \pm 0.6$ & $3.6 \pm 0.6$ & 0.168 \\
\hline TG (mmol/L) & $1.5 \pm 0.7$ & $1.6 \pm 0.8$ & 0.889 \\
\hline ALP (IU/L) & $76 \pm 21$ & $72 \pm 18$ & 0.826 \\
\hline AST (IU/L) & $36 \pm 14$ & $38 \pm 13$ & 0.381 \\
\hline ALT (IU/L) & $35 \pm 16$ & $39 \pm 22$ & 0.611 \\
\hline GGT (IU/L) & $33 \pm 19$ & $32 \pm 19$ & 0.712 \\
\hline hs-Crp (mg/L) & $4.3 \pm 7.1$ & $4.0 \pm 5.4$ & 0.865 \\
\hline Apo B (g/L) & $1.25 \pm 0.20$ & $1.25 \pm 0.21$ & 0.952 \\
\hline $\mathrm{Lp}(\mathrm{A})(\mathrm{mg} / \mathrm{dL})$ & $17 \pm 14$ & $16 \pm 16$ & 0.449 \\
\hline Serum creatinine $(\mu \mathrm{mol} / \mathrm{L})$ & $86 \pm 13$ & $78 \pm 11$ & 0.008 \\
\hline Fasting glucose (mmol/L) & $5.4 \pm 0.8$ & $6.1 \pm 2.3$ & 0.557 \\
\hline $\mathrm{IFG} \geq 7.0 \mathrm{mmol} / \mathrm{L}$ & $2(4.7 \%)$ & $7(15.9 \%)$ & \\
\hline Systolic (mmHg) & $134 \pm 18$ & $131 \pm 15$ & 0.524 \\
\hline Diastolic (mmHg) & $80 \pm 9$ & $80 \pm 9$ & 0.976 \\
\hline Tobacco, current users & $5(11.6 \%)$ & $5(11.4 \%)$ & \\
\hline Mild NAFLD (score 3) & $34(79.1 \%)$ & $35(79.5 \%)$ & \\
\hline Moderate NAFLD (score 4) & $7(16.3 \%)$ & $8(18.2 \%)$ & \\
\hline Severe NAFLD (score 5-6) & $2(4.7 \%)$ & $1(2.3 \%)$ & \\
\hline
\end{tabular}

Baseline biochemical, anthropometric and clinical parameters of all patients enrolled in the study $(n=87)$. Data are expressed as mean value \pm SD; $P<0.05$ is considered significant.

Table 2 Abbreviations: BMI Body Mass Index, TC Total Cholesterol,

HDL High Density Lipoprotein, LDL Low Density Lipoprotein,

TG Triglycerides, ALP Alkaline Phosphatase, AST aspartate transaminase,

$A L T$ alanine transaminase, GGT gamma-glutamyl transpeptidase, $h s-C R P$ high-sensitivity $C$-reactive protein, $A p o B$ apolipoprotein $B, L P(A)$ lipoprotein $A$, IFG Impaired Fasting Glucose, NAFLD Nonalcoholic Fatty Liver Disease.

[Wilcoxon rank test; Numbers' 09, GraphPad].

Conversion factor $\mathrm{mmol} / \mathrm{L}$ to $\mathrm{mg} / \mathrm{dL}$ for optimal normal values:

TC $5.2 \mathrm{mmol} / \mathrm{L}=200 \mathrm{mg} / \mathrm{dL}$;

$\mathrm{HDL} 1.55 \mathrm{mmol} / \mathrm{L}=60 \mathrm{mg} / \mathrm{dL}$;

$\mathrm{LDL} 2.6 \mathrm{mmol} / \mathrm{L}=100 \mathrm{mg} / \mathrm{dL}_{i}$

TG $1.7 \mathrm{mmol} / \mathrm{L}=151 \mathrm{mg} / \mathrm{dL}$.

normality range, with $0.63-1.33 \mathrm{~g} / \mathrm{L}$ and $0.60-1.26 \mathrm{~g} / \mathrm{L}$ for male and female respectively. Overall, blood biochemistry results indicated that tocotrienols did not significantly affect lipid profile and liver function when compared with placebo. However, a statistically significant reduction of total cholesterol $(P=0.008)$, LDL $(P=0.041)$ and TG $(P=0.027)$ in the tocotrienols group
Table 3 Intention to treat analysis of enrolled study subjects

\begin{tabular}{lcc}
\hline & \multicolumn{2}{c}{ Completion of the study } \\
\cline { 2 - 3 } Baseline $(\mathbf{n}=\mathbf{8 7})$ & Normal echogenic response & Fatty liver \\
\hline Tocotrienols $(\mathrm{n}=43)$ & $15 / 43$ & $28 / 43$ \\
& $34.9 \%$ & $65.1 \%$ \\
Placebo $(\mathrm{n}=44)$ & $8 / 44$ & $36 / 44$ \\
& $18.2 \%$ & $81.8 \%$ \\
Tocotrienols vs Placebo & $\boldsymbol{P}=\mathbf{0 . 0 3 8}$ &
\end{tabular}

Drop-out subjects were computed as having an unchanged diagnosis of fatty liver from baseline.

[Helmert-Pearson's Chi Square; Numbers'09, GraphPad].

compared to baseline was observed. Within the placebo group TG values were significantly decreased compared to baseline in similar magnitude as the treatment group $(P=0.027)$. On the other hand, reduction of total cholesterol $(P=0.210)$ and LDL $(P=0.106)$ compared to baseline were not significant.

Plasma levels of alpha-tocopherol were in the normal reported range of 5.5-18.0 $\mu \mathrm{g} / \mathrm{ml}$ [15] for all patients. The mean baseline levels of alpha-tocopherol were similar for the two groups of participants, being

Table 4 Per protocol analysis of ultrasound examination

\begin{tabular}{|c|c|c|c|c|}
\hline \multirow{2}{*}{$\begin{array}{l}\text { Tocotrienols } \\
\text { group }(n=30) \\
\text { Baseline }\end{array}$} & \multicolumn{4}{|c|}{$\begin{array}{c}\text { Changes at completion } \\
\text { of the study }\end{array}$} \\
\hline & Negative & Mild & Moderate & Severe \\
\hline Mild $(n=22)$ & $13 / 22$ & $9 / 22$ & $0 / 22$ & $0 / 22$ \\
\hline $73.3 \%$ & $59.1 \%$ & $40.9 \%$ & $0.0 \%$ & $0.0 \%$ \\
\hline Moderate $(n=6)$ & $2 / 6$ & $4 / 6$ & $0 / 6$ & $0 / 6$ \\
\hline $20.0 \%$ & $33.3 \%$ & $66.7 \%$ & $0.0 \%$ & $0.0 \%$ \\
\hline Severe $(n=2)$ & $0 / 2$ & $1 / 2$ & $0 / 2$ & $1 / 2$ \\
\hline $6.7 \%$ & $0.0 \%$ & $50.0 \%$ & $0.0 \%$ & $50.0 \%$ \\
\hline \multirow[t]{2}{*}{ TOTAL } & $15 / 30$ & $14 / 30$ & $0 / 30$ & $1 / 30$ \\
\hline & $50.0 \%$ & $46.7 \%$ & $0.0 \%$ & $3.3 \%$ \\
\hline $\begin{array}{l}\text { Placebo group } \\
(n=34)\end{array}$ & \multicolumn{4}{|c|}{$\begin{array}{l}\text { Changes at completion } \\
\text { of the study }\end{array}$} \\
\hline Baseline & Negative & Mild & Moderate & Severe \\
\hline Mild $(n=27)$ & $8 / 27$ & $17 / 27$ & $2 / 27$ & $0 / 27$ \\
\hline $79.4 \%$ & $29.6 \%$ & 63.0 & $7.4 \%$ & $0.0 \%$ \\
\hline Moderate $(n=6)$ & $0 / 6$ & $5 / 6$ & $1 / 6$ & $0 / 6$ \\
\hline $17.6 \%$ & $0.0 \%$ & $83.3 \%$ & $16.7 \%$ & $0.0 \%$ \\
\hline Severe $(n=1)$ & $0 / 1$ & $0 / 1$ & $1 / 1$ & $0 / 1$ \\
\hline $2.9 \%$ & $0.0 \%$ & $0.0 \%$ & $100.0 \%$ & $0.0 \%$ \\
\hline \multirow[t]{2}{*}{ TOTAL } & $8 / 34$ & $22 / 34$ & $4 / 34$ & $0 / 34$ \\
\hline & $23.5 \%$ & $64.7 \%$ & $11.8 \%$ & $0.0 \%$ \\
\hline $\begin{array}{l}\text { Tocotrienols } \\
\text { vs Placebo }\end{array}$ & Chi $S q=4.851$ & $P=0.014$ & & \\
\hline
\end{tabular}

Details of the clinical evaluation of subjects at baseline and relative changes after 1 year of treatment.

[Helmert-Pearson's Chi Square; Numbers'09, GraphPad]. 
Table 5 Subjects parameters changes

\begin{tabular}{|c|c|c|c|}
\hline Variable & $\begin{array}{l}\text { Tocotrienols } \\
\quad(n=30)\end{array}$ & $\begin{array}{l}\text { Placebo } \\
(n=34)\end{array}$ & $\begin{array}{c}P \text { Value } \\
\text { (Tocotrienols vs Placebo) }\end{array}$ \\
\hline $\mathrm{BMI}\left(\mathrm{kg} / \mathrm{m}^{2}\right)$ & -0.6 & -0.3 & 0.527 \\
\hline $\mathrm{TC}(\mathrm{mmol} / \mathrm{L})$ & -0.3 & -0.2 & 0.055 \\
\hline $\mathrm{HDL}$ (mmol/L) & -0.04 & 0.02 & 0.778 \\
\hline $\mathrm{LDL}(\mathrm{mmol} / \mathrm{L})$ & -0.2 & -0.1 & 0.052 \\
\hline TG (mmol/L) & -0.2 & -0.2 & 0.814 \\
\hline ALP (IU/L) & -7.0 & 1.4 & 0.471 \\
\hline AST (IU/L) & -4.8 & -3.4 & 0.207 \\
\hline $\mathrm{ALT}(\mathrm{IU} / \mathrm{L})$ & -5.9 & -0.6 & 0.118 \\
\hline GGT (IU/L) & -1.4 & -4.2 & 0.364 \\
\hline C-rp (mg/L) & -0.2 & -0.2 & 0.835 \\
\hline Apo B (g/L) & -0.01 & -0.03 & 0.046 \\
\hline $\mathrm{Lp}(\mathrm{A})(\mathrm{mg} / \mathrm{dL})$ & 2.3 & 0.4 & 0.524 \\
\hline $\begin{array}{l}\text { Serum creatinine } \\
(\mu \mathrm{mol} / \mathrm{L})\end{array}$ & -5.1 & -1.9 & 0.524 \\
\hline $\begin{array}{l}\text { Fasting glucose } \\
(\mathrm{mmol} / \mathrm{L})\end{array}$ & 0.5 & 0.5 & 0.490 \\
\hline Systolic (mmHg) & 0.1 & 0.6 & 0.660 \\
\hline Diastolic (mmHg) & -0.3 & -2.4 & 0.607 \\
\hline
\end{tabular}

Mean changes in anthropometric and laboratory parameters for the study subjects after 1 year of treatment. $P<0.05$ is considered significant. [ANOVA for split-plot design adjusted for unweighted means; Numbers'09]. Abbreviations as in Table 2.

$13.0 \pm 5.9 \mu \mathrm{g} / \mathrm{ml}$ and $14.6 \pm 6.0 \mu \mathrm{g} / \mathrm{ml}$ for placebo and tocotrienols groups, respectively. At conclusion of the study, the values for alpha-tocopherol were substantially unchanged in the placebo group with a mean concentration of $13.4 \pm 7.0 \mu \mathrm{g} / \mathrm{ml}$. Whereas, in the tocotrienol group the values of alpha-tocopherol were found to be $18.5 \pm 6.8 \mu \mathrm{g} / \mathrm{ml}$. Nevertheless, the changes in alphatocopherol levels were found to be statistically nonsignificant $(\mathrm{P}=0.665)$.

Baseline tocotrienol levels were negligible in both groups. Only alpha-tocotrienol was above the minimum limit of quantification, with $25.0 \pm 21.0 \mathrm{ng} / \mathrm{ml}$ and $26.7 \pm 32.6 \mathrm{ng} / \mathrm{ml}$ for tocotrienols and placebo group respectively. Gamma- and delta-tocotrienols were below the minimum limit of quantification in both groups.

High levels of tocotrienols were measured in all participating volunteers of the tocotrienols group at conclusion of the study, thus confirming a good level of compliance. Mean values were $212.0 \pm 379.3 \mathrm{ng} / \mathrm{ml}$, $181.4 \pm 440.8 \mathrm{ng} / \mathrm{ml}$ and $41.4 \pm 98.4 \mathrm{ng} / \mathrm{ml}$ for alpha-, gamma- and delta-tocotrienol respectively. On the other hand, they were negligible in the placebo group, with $21.7 \pm 27.9 \mathrm{ng} / \mathrm{ml}$ for alpha-tocotrienol and below the minimum limit of quantification for gamma- and deltatocotrienols.

\section{Discussion}

Vitamin E, as alpha-tocopherol has been widely investigated in liver diseases earning the status of most promising treatment at a daily dosage of $533.3 \mathrm{mg}$ (or $800 \mathrm{IU}$ ) in non-diabetics with biopsy-proven NASH [16]. The present study is the first to consider the hepatoprotective effects of tocotrienols. Their effectiveness in ameliorating NAFLD could in part be explained by the fact that the tocotrienols have been shown to distribute preferentially to the liver [6,7] where alpha-tocotrienol has been found to exert a 40-60 times more potent antioxidant activity in microsomal lipid peroxidation [8]. Oxidative stress has been recognised amongst the contributing factors in the progression of steatosis [2,17] with involvement of nuclear factor- $\kappa \mathrm{B}(\mathrm{NF}-\mathrm{k} \mathrm{B})$ activation and increased release of proinflammatory cytokines, such as tumour necrosis factor alpha (TNF $\alpha$ ) and the interleukin-6 (IL-6) representing the second insult in the "two hits" model $[18,19]$. Tocotrienols were reported to inhibit TNF $\alpha$ and to "prevent reactive oxygen species-induced NF- $\mathrm{kB}$ expression" to a higher extent compared to alpha-tocopherol in streptozotocin-treated diabetic rats [20]. Recently, biological mechanisms for the activity of tocotrienols against hepatic triglycerides accumulation have been reported. It has been shown that gamma-tocotrienol, but not alphatocopherol, was able to attenuate triglycerides accumulation by regulating fatty acid syntase and carnitine palmitoyltransferase leading to a reduction of hepatic inflammation and endoplasmic reticulum stress [21,22]. A 16-week study on rats fed high fat and high carbohydrate diet, showed a reduction of the hepatic fatty changes with "normalised portal inflammatory cells" [23]. The authors suggested that interference with the inflammatory processes to be one of the possible mechanisms for hepatoprotection [23].

Clinical trials employing the same mixed tocotrienols preparation used in the present study has been shown to be effective in improving arterial compliance [24] and in reducing Model for End-stage Liver Disease (MELD) score [7]. Moreover, the lipid lowering activity of tocotrienols has been previously reported [25] and a modest reduction of lipid profile was recorded also in the present study. Blood lipids biochemistry results showed that tocotrienols significantly reduced TC, LDL and TG compared to baseline, but not compared to placebo. However, the values for TC and LDL were still above the normality range.

In our study, baseline levels of alpha-tocopherol did not differ between the two groups. The plasma alphatocopherol concentrations of subjects in the tocotrienols treated group at conclusion of the study were found to be slightly higher than baseline, although not statistically significant. The presence of $61.1 \mathrm{mg}$ of alpha-tocopherol in the mixed tocotrienols preparation administered may 
have contributed to slight levels increase of alphatocopherol. Nevertheless, mean plasma concentrations of alpha-tocopherol in the participating subjects were still within the normal range of $5.5-18.0 \mu \mathrm{g} / \mathrm{ml}$ reported by the National Reference Laboratory [15] throughout the study, irrespective of the treatment received. The presence of alpha-tocopherol in the mixed tocotrienols capsules was unlikely to have any influence on the outcomes of the present study since the amount present in the capsules was far below those used in earlier studies with negative outcomes [26-29].

We acknowledge that histology examination is the gold standard for evaluation of liver diseases and the use of USG might represent an intrinsic limitation. However, USG examination has been shown to have adequate sensitivity and specificity, in particular as front line evaluation of non-advanced fatty liver.

Diagnostic reports showed that USG has specificity range of $60-100 \%$ and sensitivity range of $80-100 \%$ [30-32]. Obesity with BMI above 40 might affect USG evaluation, reducing specificity and sensitivity when fat infiltration is below 33\% [33]. In the present study, only one morbid obese was enrolled whose baseline diagnosis of NAFLD was unchanged at conclusion.

A gross advantage of USG is that the liver is seen in full, while a needle biopsy only samples about one $50,000^{\text {th }}$ of the organ and area sampling error could be an issue in non-advanced cases [31,34]. The choice of investigating hepatoprotective activity of a nutritional supplement, such as mixed tocotrienols, in adults drawn from a population-based study [10] with the use of noninvasive USG has to be seen in this light, being the majority of patients classified as mild steatosis with normal to slightly elevated liver aminotransferases, thus a non-advanced stage of NAFLD.

A possible weakness of our study may lie in the use of USG versus other non-ionising imaging methodologies such as proton magnetic resonance spectroscopy $\left({ }^{1} \mathrm{H}\right.$ MRS). ${ }^{1} \mathrm{H}$ MRS is a powerful and objective tool for assessing fat fraction in the liver, even though it lacks of "information about regional fat distribution" [35].

A recent study [36] aimed at correlating ${ }^{1} \mathrm{H}$ MRS with the echodiscrepancy ratio between kidney and liver as indicator of steatosis, on subjects with essentially similar anthropometric and blood biochemical values to our present study, concluded that the hepatorenal echodiscrepancy ratio evaluated with USG is in accord with the quantitative data obtained from ${ }^{1} \mathrm{H}$ MRS for hepatic lipid infiltration in the range 1\%-30\% for comparable severity of steatosis, albeit what Mancini [36] considered as moderate in their study, is categorised as mild in ours.

We acknowledge that our patients were a low-risk cohort. However, patients with similar characteristics as those considered in our study, including normal liver transaminases levels, have been shown to still be at risk with respect to progression of NAFLD $[37,38]$.

In view of the not fully elucidated reasons for steatosis to progress to more advanced stages of liver disease, it might be beneficial to manage the condition during the early stages, such as at the steatotic stage. Hence, in view of our findings, mixed tocotrienols might be considered for the treatment of steatosis.

In summary, the present study showed that patients assigned to tocotrienols at the current dosage, have significantly benefited in terms of normalisation of the hepatic echogenic response in NAFLD compared to placebo during the 1-year treatment. We acknowledge that this is a pilot study and therefore larger cohort studies should be warranted to further confirm the positive results of tocotrienols in NAFLD of the present study. Furthermore, at the current combined dosage of $400 \mathrm{mg} / \mathrm{d}$ used in the present study, the mixed tocotrienols were well tolerated and no adverse reactions were reported.

\section{Abbreviations}

NAFLD: Non-alcoholic fatty liver disease; NASH: Non-alcoholic steatohepatitis; BMI: Body mass index; TC: Total cholesterol; HDL: High density lipoprotein; LDL: Low density lipoprotein; TG: Triglycerides; ALP: Alkaline phosphatase; AST: Aspartate transaminase; ALT: Alanine transaminase; GGT: Gammaglutamyl transpeptidase; hs-CRP: High-sensitivity C-reactive protein; ApoB: Apolipoprotein B; LP(A): Lipoprotein A; IFG: Impaired fasting glucose; HPLC: High-performance liquid chromatography; USG: Ultrasonography; Cl: Confidence interval; NNT: Number needed to treat; OR: Odds ratio; NF-KB: Nuclear factor-KB; TNFa: Tumour necrosis factor alpha;

IL-6: Interleukin-6; ${ }^{1}$ H MRS: Proton magnetic resonance spectroscopy; HCC: Hepatocellular carcinoma.

\section{Competing interests}

JW Wong, BH Ng, KH Yuen contributed a chapter ("Absorption and disposition of tocotrienols") in the book: "Tocotrienols: vitamin E beyond tocopherols" Watson\&Preedy Eds, CRC Press, Boca Raton, FL, USA. KH Yuen has patents related to the investigational product (self-microemulsifying delivery system for fat-soluble drugs: US patent 6596306). KH Yuen is an independent consultant for Hovid. KH Yuen, JW Wong, BH Ng \& E Magosso own shares of Hovid. None of the authors is a substantial shareholder of Hovid. E Magosso, KH Yuen \& IL Shuaib filed a patent application for the indication of tocotrienols in prevention of liver diseases. KH Yuen \& $E$ Magosso have served as speakers for Hovid, but without receiving any financial remuneration or gratuity. Y Gopalan, MA Ansari, NAK Khan, MR Abu Bakar have no potential conflict of interest.

\section{Authors' contributions}

Guarantors for the article: KHY \& EM. ILS, MAA performed the Radiological Investigations and Clinical Evaluations; MRAB contributed to the Radiological Investigations and Clinical Evaluations. EM, KHY, ILS and NAKK designed the research protocol and wrote the manuscript; EM and YG collected and analysed the data; JWW was responsible for the randomisation; BHN contributed to the randomisation. All authors read and approved the final manuscript.

\section{Acknowledgements}

The authors are thankful to Dr K Nesaretnam, director of Product Development Research \& Advisory Services of MPOB, for her support throughout the study. 


\section{Financial support}

The authors acknowledge the Malaysian Palm Oil Board (MPOB) for providing the supporting research grant. E Magosso is the recipient of an Incentive Grant for NAFLD by Universiti Sains Malaysia.

\section{Author details}

${ }^{1}$ Advanced Medical and Dental Institute, Universiti Sains Malaysia, Kepala Batas, Malaysia. ${ }^{2}$ School of Pharmaceutical Sciences, Universiti Sains Malaysia, Penang, Malaysia. ${ }^{3}$ Faculty of Pharmacy, Universiti Teknologi MARA, Puncak Alam, Malaysia. ${ }^{4}$ Hovid Research Sdn Bhd, Penang, Malaysia.

Received: 22 July 2013 Accepted: 12 December 2013

Published: 27 December 2013

\section{References}

1. Letteron P, Fromenty B, Terris B, Degot C, Pessayre D: Acute and chronic hepatic steatosis lead to in vivo lipid peroxidation in mice. $J$ Hepatol 1996, 24:200-208.

2. Albano E, Mottaran E, Occhino G, Reale E, Vidali M: Review article: role of oxidative stress in the progression of non-alcoholic steatosis. Aliment Pharmacol Ther 2005, 22(Suppl. 2):71-73.

3. Allard JP, Aghdassi E, Mohamed S, Raman M, Avand G, Arendt BM, et al: Nutritional assessment and hepatic fatty acid composition in non-alcoholic fatty liver disease (NAFLD): a cross-sectional study. J Hepatol 2008, 48:300-307.

4. Sanyal AJ, Chalasani N, Kowdley KV, McCullogh A, Diehl AM, Bass NM, et al: Pioglitazone, vitamin $\mathrm{E}$, or placebo for nonalcoholic steatohepatitis. N Engl J Med 2010, 362(18):1675-1685.

5. Lavine JE, Schwimmer JB, Van Natta ML, Molleston JP, Murray KF, Rosenthal $P$, et al: Effects of vitamin $E$ or metformin for treatment of nonalcoholic fatty liver disease in children and adolescents. JAMA 2010, 305(16):1659-1668.

6. Yuen $\mathrm{KH}, \mathrm{Ng} \mathrm{BH}$, Wong JW: Absorption and disposition of tocotrienols. In Tocotrienols: vitamin E beyond tocopherols. Edited by Watson RR, Preedy VR. Boca Raton: CRC Press; 2009:297-308.

7. Patel V, Rink C, Gordillo GM, Khanna S, Gnyawali U, Roy S, et al: Oral tocotrienols are transported to human tissues and delay the progression of the model for end-stage liver disease score in patients. J Nutr 2012, 142:513-519.

8. Serbinova E, Kagan V, Han D, Packer L: Free radical recycling and intramembrane mobility in the antioxidant properties of alphatocopherol and alpha-tocotrienol. Free Radic Biol Med 1991, 10:263-275.

9. Farrell GC, Wong WWS, Chitturi S: NAFLD in ASIA - as common and important as in the west. Nat Rev Gastroenterol Hepatol 2013, 10:307-318.

10. Magosso E, Ansari MA, Yogheswaran G, et al: Prevalence of non-alcoholic fatty liver in a hypercholesterolemic population of northwestern peninsular Malaysia. Southeast Asian J Trop Med Public Health 2010, 41(4):936-942.

11. Yap SP, Julianto T, Wong JW, Yuen KH: Simple high-performance liquid chromatographic method for the determination of tocotrienols in human plasma. J Chromatogr B 1999, 735:279-283.

12. Tominaga $\mathrm{K}$, Kurata $J \mathrm{H}$, Chen $\mathrm{YK}$, et al: Prevalence of fatty liver in Japanese children and relationship to obesity. Dig Dis Sci 1995, 40(9):2002-2009.

13. Pocock SJ: Clinical trials: a practical approach. Chicester: John Wiley\&Sons; 1983.

14. Kirk RE: Experimental design procedures for the behavioral sciences. Pacific Grove: Brooks/Cole; 1968

15. ARUP National Reference Laboratory: [http://www.aruplab.com/guides/ug/ tests/0080521.jsp]

16. Chalasani N, Younossi Z, Lavine JE, Diehl AM, Brunt EM, Cusi K, et al: The diagnosis and management of non-alcoholic fatty liver disease: practice guideline by the American association for the study of liver diseases, American college of gastroenterology, and American gastroenterological association. Hepatology 2012, 55(6):2005-2023.

17. Begriche $K$, Igoudjil A, Pessaryre D, Fromenty B: Mitochondrial dysfunction in NASH: causes, consequences and possible means to prevent it. Mitochondrion 2006, 6:1-28.

18. Day CP: Pathogenesis of steatohepatitis. Best Pract Res Clin Gastroenterol 2002, 16(5):663-678.

19. Farrell GC, Larter CZ: Nonalcoholic fatty liver disease: from steatosis to cirrhosis. Hepatology 2006, 43:S99-S112.
20. Kuhad A, Chopra K: Attenuation of diabetic nephropathy by tocotrienol: involvement of NFkB signalling pathway. Life Sci 2009, 84:296-301.

21. Burdeos GC, Nakagawa K, Watanabe A, Kimura F, Miyazawa T: Gammatocotrienol attenuates triglyceride through effect on lipogenic gene expression in mouse hepatocellular carcinoma Hepa 1-6. J Nutr Sci Vitaminol 2013, 59:148-151.

22. Muto C, Yachi R, Aoki Y, Koike T, Igarashi O, Kiyose C: Gamma-tocotrienol reduces the triacylglycerol level in rat primary hepatocytes through regulation of fatty acid metabolism. J Clin Biochem Nutr 2013, 52(1):32-37.

23. Wong WY, Poudyal H, Ward LC, Brown L: Tocotrienols reverse cardiovascular, metabolic and liver changes in high carbohydrate, high fat diet-fed rats. Nutrients 2012, 4:1527-1541.

24. Rasool AH, Rahman AR, Yuen KH, Wong AR: Arterial compliance and vitamin $E$ blood levels with a self emulsifying preparation of tocotrienol rich vitamin E. Arch Pharm Res 2008, 31(9):1212-1217.

25. Baliarsingh S, Beg ZH, Ahmad J: The therapeutic impacts of tocotrienols in type 2 diabetic patients with hyperlipidemia. Atherosclerosis 2005 , 182:367-374.

26. Hasegawa T, Yoneda M, Nakamura K, Makino I, Terano A: Plasma transforming growth factor- $\beta 1$ level and efficacy of a-tocopherol in patients with non-alcoholic steatohepatitis: a pilot study. Aliment Pharmacol Ther 2001, 15:1667-1672.

27. Harrison SA, Torgerson S, Hayashi P, Ward J, Schenker S: Vitamin E and vitamin $C$ treatment improves fibrosis in patients with nonalcoholic steatohepatitits. Am J Gastroenterol 2003, 98:2485-2490.

28. Kugelmas M, Hill DB, Vivian B, Marsano L, McClain CJ: Cytokines and NASH: a pilot study of the effects of lifestyle modification and vitamin $\mathrm{E}$. Hepatology 2003, 38(2):413-419.

29. Vajro P, Mandato C, Franzese A, Ciccimarra E, Lucariello S, Savoia M, et al: Vitamin E treatment in pediatric obesity-related liver disease: a randomized study. J Pediatr Gastroenterol Nutr 2004, 38:48-55.

30. Palmenteri B, de Sio I, La Mura V, Masarone M, Vechione R, Bruno S, et al: The role of bright liver echo pattern on ultrasound B-mode examination in the diagnosis of liver steatosis. Dig Liver Dis 2006, 38:485-489.

31. Metha SR, Thomas EL, Bell JD, Johnston DG, Taylor-Robinson SD: Non-invasive means of measuring hepatic fat content. World J Gastroenterol 2008, 14(22):3476-3483.

32. Dasarathy S, Dasarathy J, Khiyami A, Joseph R, Lopez R, McCullogh AJ: Validity of real time ultrasound in the diagnosis of hepatic steatosis: a prospective study. J Hepatol 2009, 51:1061-1067.

33. Saadeh S, Younossi ZM, Remer EK, Gramlich T, Ong J, Hurley M, et al: The utility of radiological imaging in nonalcoholic fatty liver disease. Gastroenterology 2002, 123:745-750.

34. Brunt EM, Tiniakos DG: Histopathology of nonalcoholic fatty liver disease. World J Gastroenterol 2010, 16(42):5286-5296.

35. Schwenzer NF, Springer F, Schraml C, Stefan N, Machmann J, Schick F: Non-invasive assessment and quantification of liver steatosis by ultrasound, computed tomography and magnetic resonance. $J$ Hepatol 2009, 51:433-445.

36. Mancini M, Prinster A, Annuzzi G, Liuzzi R, Giacco R, Medagli C, et al: Sonographic hepatic-renal ratio as indicator of hepatic steatosis: comparison with ${ }^{1} \mathrm{H}$ magnetic resonance spectroscopy. Metabolism 2009, 58:1724-1730.

37. Mofrad P, Contos MJ, Haque M, Sargeant C, Fisher RA, Luketic VA, et al: Clinical and histologic spectrum of nonalcoholic fatty liver disease associated with normal ALT values. Hepatology 2003, 37:1286-1292.

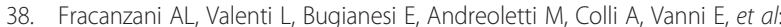
Risk of severe liver disease in nonalcoholic fatty liver disease with normal aminotransferase levels: a role for insulin resistance and diabetes. Hepatology 2008, 48:792-798.

doi:10.1186/1475-2891-12-166

Cite this article as: Magosso et al:: Tocotrienols for normalisation of hepatic echogenic response in nonalcoholic fatty liver: a randomised placebo-controlled clinical trial. Nutrition Journal 2013 12:166. 